\title{
Effect of controlling maximum-SNR-based relay selection strategy in cooperative wireless communication systems
}

\author{
Essam Saleh Altubaishi \\ Department of Electrical Engineering, King Saud University, Saudi Arabia
}

\begin{tabular}{|c|c|}
\hline Article Info & ABSTRACT \\
\hline Article history: & Relay selection strategy under maximum-signal-to-noise ratio (MAX-SNR) \\
\hline Received Oct 15, 2020 & $\begin{array}{l}\text { criterion was proven to maximize performance but at the expense of losing } \\
\text { fairness among the cooperative relays. In this work, the effect of controlling }\end{array}$ \\
\hline Revised Jan 26, 2021 & the MAX-SNR criterion on the spectral efficiency of cooperative wireless \\
\hline Accepted Feb 15, 2021 & $\begin{array}{l}\text { communication system with adaptive modulation is investigated. } \\
\text { Specifically, the probability density function (PDF) of the end-to-end SNR }\end{array}$ \\
\hline Keywords: & $\begin{array}{l}\text { for the considered system is derived when the controlled selection criterion is } \\
\text { considered. Base on that PDF, the average spectral efficiency is then derived }\end{array}$ \\
\hline $\begin{array}{l}\text { Adaptive modulation } \\
\text { Amplify-and-forward } \\
\text { Cooperative diversity }\end{array}$ & $\begin{array}{l}\text { and investigated. The results show how the spectral efficiency of the system } \\
\text { deteriorates as controlling the selection of a relay. Furthermore, the results of } \\
\text { Monte Carlo simulation validate the derived expression. }\end{array}$ \\
\hline
\end{tabular}

This is an open access article under the CC BY-SA license.

\begin{abstract}
Relay selection strategy under maximum-signal-to-noise ratio (MAX-SNR) criterion was proven to maximize performance but at the expense of losing fairness among the cooperative relays. In this work, the effect of controlling Specifically, the probability density function (PDF) of the end-to-end SNR for the considered system is derived when the controlled selection criterion is and investigated. The results show how the spectral efficiency of the system Monte Carlo simulation validate the derived expression.
\end{abstract}

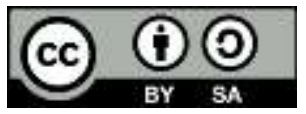

\section{Corresponding Author:}

Essam Saleh Altubaishi

Department of Electrical Engineering

King Saud University

Riyadh, 11421, Saudi Arabia

Email: etubashi@ksu.edu.sa

\section{INTRODUCTION}

Cooperative wireless communication has emerged as an effective technique that provides high communication reliability, increased throughput, and better coverage area. However, it has undesirable feature which is increasing number of transmission channels as number of relays increases. Relay selection represents an important technique to solve this problem in which one or subgroup of the relays is selected out of group of relays based on a pre-defined criterion [1-7].

Various relay selection schemes with different objectives have been proposed [8-13]. Opportunistic relaying (OR) was introduced in [8] in which a single relay node is selected opportunistically. This scheme is characterized by simple process which can be implemented easily in existing wireless systems. In [9], we introduce an amplify-and-forward (AF) adaptive relaying scheme with relay selection strategy based on OR and variable-rate transmission. The results indicated that the proposed scheme outperformed fixed relaying scheme especially at low values of signal-to-noise ratio (SNR). In [10], the transmitting power was optimized when OR is exploited in sensor networks. In [11], outage probability is derived for AF OR under high-SNR scenario. Same system model with same performance metric considered in [11] was investigated in [12] but with a decode-and-forward (DF) relaying. The authors of [13] extended the results of [12] by studying the effect of channel state information (CSI) on the average symbol error probability.

Other research works have included other factors, such as constraint on the resources of the relay node [14-24]. In [14], a relay selection method was proposed to consider the power consumption of each relay node during helping other nodes. An opportunistic relay selection method was introduced in [15] where 
the achieved fairness among the relay nodes for a given throughput was only investigated. The work of [16] combined the spectral efficiency of successive opportunistic relaying with the strength of single-link relay selection in order to reduce the energy consumption per time slot. Whereas $[17,18]$ combined the frame aggregation with the channel condition in the selection criteria to improve transmission rate. Another method called modified adaptive best relay selection was introduced and simulated in [20]. The simulation results verified that the energy efficiency could be improved by utilizing the proposed selection criterion. The authors of [21] proposed an incentive mechanism to motivate a node to act as a relay. The benefit from relaying can be reduction of energy consumption, enhancement of capacity, or both. In [22], a single-relay selection method for cognitive cooperative networks was introduced under interference and power constraints. The results demonstrated that this method is able to improve the bandwidth efficiency. In [23], a relay selection strategy was proposed to consider the buffer state of the relay node. The results of the proposed strategy confirmed that better outage and packet-delay performance could be achieved. The authors of [24] also proposed another relay selection method using maximum minimum distance criterion to improve sum-rate, average delay, and bit error rate.

One can notice that the works $[14,16,18,20-22]$ investigated the performance using simulation only, and all the works [14-24] did not derive closed-form expression for the performance measure as a function of the controlling parameter of the relay selection criterion. Therefore, the objective of this work is to include the controlling parameter in the expression of the performance measure so that the performance can be investigated under controlled relay selection criterion. In this work, AF parallel relaying system with relay selection and adaptive modulation is considered. The average spectral efficiency is derived as a function not only of the average SNR of the existing links but also the controlling parameter used to control the relay selection. The derived expression can be achieved by deriving first the PDF of the end-to-end SNR of the considered system. Finally, a Monte Carlo simulation is used to verify the derivation.

The remainder of this paper is organized as follows. System model is presented in Section 2. Controlling the selection of a relay node is presented in Section 3. Probability density function (PDF) of the end-to-end SNR is derived in Section 4 in addition to the derivation of a closed-form expression for the average spectral efficiency. In Section 5, some numerical examples are presented to evaluate our proposed strategy. Finally, conclusion of the paper is given in Section 6.

\section{SYSTEM OVERVIEW}

An AF cooperative wireless system with relay selection and adaptive modulation is considered which consists of 2 relays $\left(r_{i}, i=1,2\right)$. Only one relay is selected to forward the received information from source s to destination $d$. All links are modeled as Rayleigh fading channels. Also, the selection of the relay needs a local knowledge of the instantaneous SNR of the relay path.

The transmission begins with transmitting the source information $I(t)$ to both the selected relay $r_{b}$ and the destination $d$. The received signals at $r_{b}$ and $d$ can be written as (1) and (2)

$$
\begin{gathered}
y_{s r_{b}}(t)=g_{s r_{b}} I(t)+n_{s r_{b}}(t), \\
y_{s d}(t)=g_{s d} I(t)+n_{s d}(t),
\end{gathered}
$$

respectively, where $\mathrm{g}_{s r_{b}}$ and $\mathrm{g}_{s d}$ are the fading coefficients of $s-r$ link and $s-d$ link, respectively. $n_{s r_{b}}(t)$ and $n_{s d}(t)$ are the Additive White Gaussian Noise (AWGN) terms at $r_{b}$ and $d$, respectively, with a variance of No. $r_{b}$ then amplifies the received signal $y_{s r_{b}}(t)$, and transmits it to $d$, in which the received signal becomes (3)

$$
y_{r_{b} d}(t)=G_{r_{b}} g_{r_{b} d} y_{s r_{b}}(t)+n_{r_{b} d}(t)
$$

where $\mathrm{g}_{r_{b}}$ is the fading coefficient of $r_{b}-d$ link, $n_{r_{b} d}(t)$ is the AWGN term at $d$, and $G_{r_{b}}$ is the amplifying gain which can be given as (4)[2]

$$
G_{r_{b}}^{2}=\frac{P_{r}}{P_{S}\left|g_{s r_{b}}\right|^{2}+N_{o}}
$$

where $P_{s}$ and $P_{r}$ are the transmitted powers of $s$ and $r_{b}$, respectively. The end-to-end SNR associated with the $i^{\text {th }}$ relay node is $(5)$ 


$$
\gamma_{r_{i}}=\frac{\gamma_{s r_{i}} \gamma_{r_{i} d}}{\gamma_{s r_{i}}+\gamma_{r_{i}} d+1}
$$

where $\gamma_{s_{i}}$ and $\gamma_{r_{i} d}$ are the instantaneous SNRs between $s$ and the $i^{\text {th }}$ relay, and the $i^{\text {th }}$ relay and $d$, respectively. To simplify the derivation, (5) can be approximated by its upper bound as $(6)[9,10]$

$$
\gamma_{r_{i}} \leq \gamma_{s r_{i} d}=\min \left(\gamma_{s r_{i}}, \gamma_{r_{i} d}\right)
$$

For the Rayleigh fading channels, the Cumulative Distribution Function (CDF) and PDF of the received SNR of any link are given by (7) and (8)

$$
\begin{aligned}
& F_{\gamma_{i j}}(x)=1-e^{-\frac{x}{\bar{\gamma}_{i j}}}, \\
& f_{\gamma_{i j}}(x)=\frac{1}{\bar{\gamma}_{i j}} e^{-\frac{x}{\bar{\gamma}_{i j}}}
\end{aligned}
$$

where $\bar{\gamma}_{i j}=E\left[\gamma_{i j}\right]$, and $\mathrm{E}[$.$] is a notation for expectation operation.$

\section{CONTROLLING RELAY SELECTION}

In general, relay selection method may use CSI, performance metrics, or both to define the selection criterion. MAX-SNR-based relay selection strategy uses only CSI, in which the best relay $r_{b}$ is selected if its bath has the largest instantaneous SNR. It is feasible to use a controlling parameter $c_{i}$ in order to control the selection criterion. Mathematically, the modified selection criterion can be represented as (9)

$$
b=\arg \max _{i=1,2}\left(\gamma_{c_{i}}\right)
$$

where $\gamma_{c_{i}}=\frac{\gamma_{r_{i}}}{c_{i}}$ is the controlled end-to-end SNR of the relay path. The output SNR at $d$ becomes (10)

$$
\gamma_{A F, b}=\gamma_{s d}+\gamma_{r_{b}}
$$

\subsection{Mode of operation}

One way to implement the modified selection criterion is to use control signaling to estimate the end-to-end SNR for each path. The source node first broadcasts a signal to the relay and destination nodes where each relay node estimates the SNR of the $s-r$ link. Then, the destination node replies by sending a signal to the relay nodes. The relay node then estimates the SNR of the $r$ - $d$ link and calculates the controlled end-to-end SNR $\gamma_{r_{i}}$, where the contolling parameter $c_{i}$ is stored in each relay node. Each relay node then calculates the backoff time $t_{i}$ required to respond to the source node. This backoff time is inversely proportional to $\gamma_{r_{i}}$ in which the relay node, with the largest $\gamma_{r_{i}}$ and the minimum $t_{i}$, will broadcast a flag containing its relay index to the relay and source nodes. Eventually, the other relay nodes will remain silent and the source node will start transmission of information to the selected relay. Note that the source node is able to change $c_{i}$ depending on the current condition of the network.

\section{AVERAGE SPECTRAL EFFICIENCY}

The average spectral efficiency of the considered system can be expressed as:

$$
\eta=\sum_{n=1}^{N} \frac{n}{2} \phi(n)
$$

where $N$ represents the number of modulation modes used, and $\phi(n)$ is the probability that the received SNR falls in region $n$ which can be defined as,

$$
\phi(n)=\int_{\gamma_{n}}^{\gamma_{n+1}} f_{\gamma_{A F, b}}(x) d x,
$$

where $\gamma_{n}$ and $\gamma_{n+1}$ represent the SNR thresholds of the $n^{\text {th }}$ region, and $f_{A F, b}(\gamma)$ is the PDF of the received SNR of selected relay node which can be written as (13), 


$$
f_{\gamma_{A F, b}}(\gamma)=\int_{0}^{\gamma} f_{\gamma_{s r_{b} d}}(\gamma-x) f_{\gamma_{s d}}(x) d x,
$$

where $f_{\gamma_{s r_{b} d}}(\gamma)$ is the PDF of the selected relay given by (14),

$$
f_{\gamma_{s r_{b} d}}(\gamma)=f_{\gamma_{s r_{1} d}}(\gamma) F_{\gamma_{s r_{2} d}}\left(\frac{c_{2}}{c_{1}} \gamma\right)+f_{\gamma_{s r_{2} d}}(\gamma) F_{\gamma_{s r_{1} d}}\left(\frac{c_{1}}{c_{2}} \gamma\right),
$$

where $F_{\gamma_{s r_{i} d}}(\gamma)$ and $f_{\gamma_{s r_{i} d}}(\gamma)$ are the CDF and PDF of the relay path given as (15) and (16),

and

$$
F_{\gamma_{s r_{i} d}}(\gamma)=1-\left(1-F_{\gamma_{s r_{i}}}(\gamma)\right)\left(1-F_{\gamma_{r_{i}}}(\gamma)\right)=1-e^{-\frac{\gamma}{\bar{\gamma}_{s r_{i}} d}},
$$

$$
f_{\gamma_{s r_{i} d}}(\gamma)=\frac{1}{\bar{\gamma}_{s r_{i} d}} e^{-\frac{\gamma}{\bar{\gamma}_{s r_{i} d}}}
$$

$\bar{\gamma}_{s r_{i} d}=\bar{\gamma}_{s r_{i}} \cdot \bar{\gamma}_{r_{i} d} /\left(\bar{\gamma}_{s r_{i}}+\bar{\gamma}_{r_{i} d}\right)$, and $\bar{\gamma}_{s r_{i}}$ and $\bar{\gamma}_{r_{i} d}$ are the average SNRs of the $s-r$ link and $r-d$ link, respectively. Substituting (8), (14), (15), and (16) into (13) then solving the integral, the PDF of the end-toend SNR of the selected relay path can be given as (17),

$$
\begin{aligned}
& f_{\gamma_{A F, b}}(\gamma)=\frac{1}{\tilde{\gamma}_{s r_{1} d}-\bar{\gamma}_{s d}} e^{-\frac{\gamma}{\bar{\gamma}_{s r_{1} d}}}-\frac{1}{\tilde{\gamma}_{s r_{1} d}-\bar{\gamma}_{s d}-\frac{c_{2} \tilde{\gamma}_{s r_{1} d} \tilde{\gamma}_{s d} \bar{\gamma}_{1} \bar{\gamma}_{s r_{2} d}}{s}} e^{-\gamma\left(\frac{1}{\bar{\gamma}_{s r_{1} d} d}+\frac{c_{2}}{c_{1} \tilde{\gamma}_{s r_{2} d}}\right)} \\
& \times \frac{1}{\tilde{\gamma}_{s r_{2} d}-\bar{\gamma}_{s d}} e^{-\frac{\gamma}{\bar{\gamma}_{s r_{2} d}}}-\frac{1}{\bar{\gamma}_{s r_{2} d}-\bar{\gamma}_{s d}-\frac{c_{1} \tilde{\gamma}_{s r_{2} d} \tilde{\gamma}_{2} \bar{\gamma}_{s r_{1} d}}{c_{s d}}} e^{-\gamma\left(\frac{1}{\bar{\gamma}_{s r_{2} d}}+\frac{c_{1}}{c_{2} \tilde{\gamma}_{s r_{1} d}}\right)}
\end{aligned}
$$

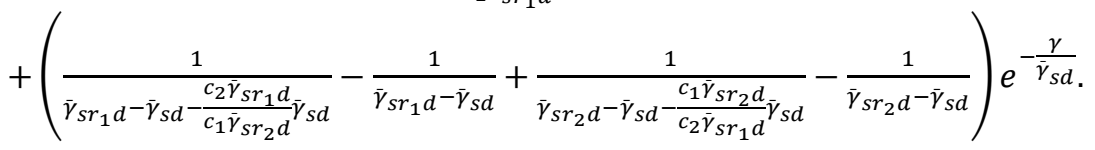

Solving the integral of (12) after utilizing the expression of $f_{\gamma_{A F, b}}(\gamma)$, the average spectral efficiency can be obtained as (18),

$$
\begin{aligned}
& \eta=\sum_{n=1}^{N} \frac{n}{2}\left(\frac{\bar{\gamma}_{s r_{1} d}}{\bar{\gamma}_{s r_{1} d}-\bar{\gamma}_{s d}}\left(e^{-\frac{\gamma_{n}}{\hat{\gamma}_{s r_{1} d}}}-e^{-\frac{\gamma_{n+1}}{\bar{s}_{s r_{1} d}}}\right)+\frac{\bar{\gamma}_{s r_{2} d}}{\bar{\gamma}_{s r_{2} d}-\bar{\gamma}_{s d}}\left(e^{-\frac{\gamma_{n}}{\hat{\gamma}_{s r_{2} d}}}-e^{-\frac{\gamma_{n+1}}{\bar{\gamma}_{s r_{2} d}}}\right)\right.
\end{aligned}
$$

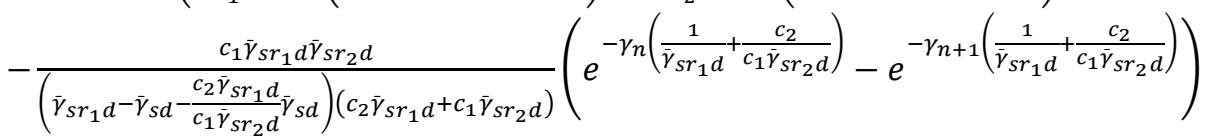

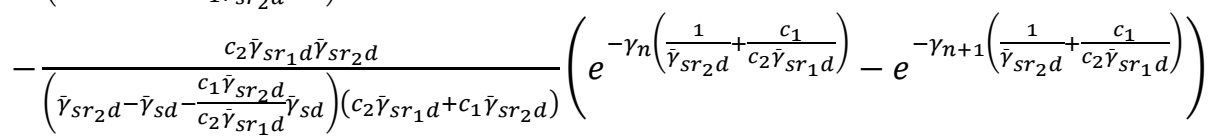

$$
\begin{aligned}
& \left.+\bar{\gamma}_{s d}\left(\frac{1}{\bar{\gamma}_{s r_{1} d}-\bar{\gamma}_{s d}-\frac{c_{2} \tilde{\gamma}_{s r_{1} d} \bar{c}_{1} \tilde{\gamma}_{s r_{2} d}}{\bar{\gamma}_{s d}}}-\frac{1}{\bar{\gamma}_{s r_{1} d}-\bar{\gamma}_{s d}}+\frac{1}{\bar{\gamma}_{s r_{2} d}-\bar{\gamma}_{s d}-\frac{c_{1} \tilde{\nu}_{s r_{2} d} \bar{\gamma}_{2} \bar{\gamma}_{s r_{1} d}}{c_{s}}}-\frac{1}{\bar{\gamma}_{s r_{2} d}-\bar{\gamma}_{s d}}\right) \times\left(e^{-\frac{\gamma_{n}}{\bar{\gamma}_{s d}}}-e^{-\frac{\gamma_{n+1}}{\bar{\gamma}_{s d}}}\right)\right)
\end{aligned}
$$

Note that the SNR thresholds for the modulation modes used can be obtained by using the bit error rate expressions for binary phase shift keying and M-ary QAM over AWGN channel [25], i.e.,

$$
\begin{aligned}
& \gamma_{1}=\left(\operatorname{erfc}^{-1}\left(2 B E R_{T}\right)\right)^{2}, n=1 \\
& \gamma_{n}=-\frac{2}{3}\left(2^{n}-1\right) \ln \left(5 B E R_{T}\right), n=0,2,3, \ldots, N \\
& \gamma_{N+1}=+\infty
\end{aligned}
$$

where $B E R_{T}$ is the target bit error rate . 


\section{NUMERICAL AND SIMULATION EXAMPLES}

In this section, numerical examples are presented to evaluate the performance of the considered system under controlled relay selection. Table 1 shows the parameters used in this investigation where we assume that relay 1 has better channel condition. Also the target bit error rate $B E R_{T}$ is assumed to be equal to $10^{-3}$, and the number of modulation modes $N=6$. For the Monte Carlo simulator, 200,000 samples are generated for the instantaneous SNR for all links of the system, this includes the direct link and all relay links from source to relay and relay to destination. For each iteration, the selection criterion with controlling parameter is calculated as in (9), and the output SNR at destination is calculated based on (10). Then the instantaneous spectral efficiency can be found by determining the region that the output SNR falls in, which can be generated by (19). After completing all iteration, the average spectral efficiency is calculated by dividing the sum of the instantaneous spectral efficiency over the number of samples.

Table 1. Channel parameters for each link.

\begin{tabular}{ccc}
\hline Average SNR & Relay 1 & Relay 2 \\
\hline $\bar{\gamma}_{s r_{i}}$ & $7.2 \bar{\gamma}_{s d}$ & $6.1 \bar{\gamma}_{s d}$ \\
$\bar{\gamma}_{r_{i} d}$ & $5.1 \bar{\gamma}_{s d}$ & $2.7 \bar{\gamma}_{s d}$ \\
\hline
\end{tabular}

Figure 1 shows the average spectral efficiency of the AF cooperative wireless communication system with relay selection and adaptive modulation versus the average SNR of the direct link. Since the first relay has better SNR, it is expected that it will be selected more frequently. To control this selection, 3 Cases are considered. Case 1 represents the reference case in which there is no control over the selection (i.e., $c_{1}=1$ and $\left.c_{2}=1\right)$, Case 2 has $\left(c_{1}=5\right.$ and $\left.c_{2}=1\right)$, and Case 3 has $\left(c_{1}=20\right.$ and $\left.c_{2}=1\right)$. It is observed that as the link quality improves the spectral efficiency increases until approaches the efficiency of the highest modulation mode used which is in this investigation is 6 . Also, it is noticed that Case 1, as expected, has the best spectral efficiency which represents the use of MAX-SNR criterion without any control. As the controlling parameter increases for Relay 1, the frequent selection will be less and hence the average spectral efficiency starts to deteriorate. One can compromise between the excessive use of a particular relay and achieving an acceptable spectral efficiency.

Figure 2 shows the average spectral efficiency of the considered system versus the average SNR of the direct link but with a reverse controlling parameters (i.e., Relay 1 has constant controlling parameter of 1 , and Relay 2 has $\left.c_{2}=[1,5,20]\right)$. It is obvious that the reduction of the spectral efficiency is less in this case. For example, when $\bar{\gamma}_{s d}=10 \mathrm{~dB}$, and Case 1 and Case 3 are considered in both figures, the average spectral efficiency of Figure 1 decreases by $17 \%$, whereas in Figure 2, the average spectral efficiency decreases by only $8 \%$.

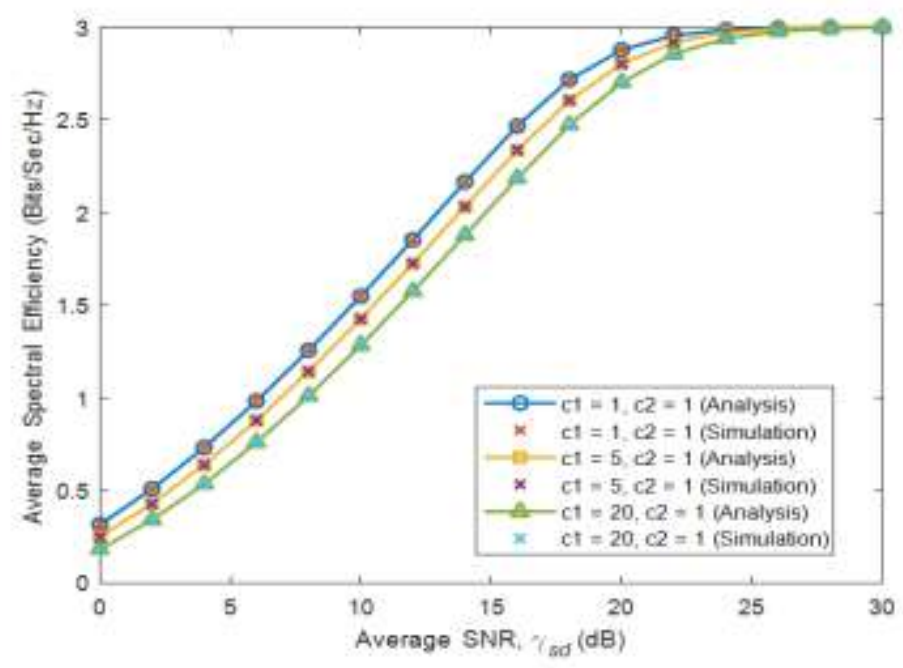

Figure 1. Average spectral efficiency of the AF cooperative wireless communication system with relay selection and amplitude modulation and different controlling parameters. 


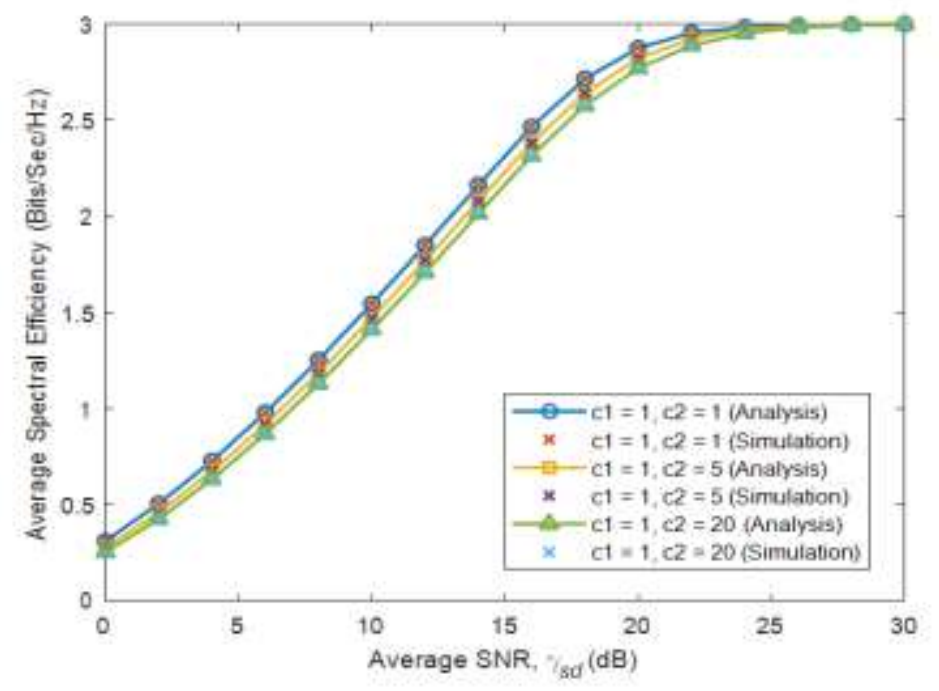

Figure 2. Average spectral efficiency of the AF cooperative wireless communication system with relay selection and amplitude modulation and reverse values of controlling parameters.

\section{CONCLUSION}

In this paper, we studied the effect of controlling the relay selection process on the spectral efficiency of the AF cooperative wireless communication systems with adaptive modulation. The PDF of the end-to-end SNR of the considered system was derived to be able to obtain a closed-form expression for the average spectral efficiency. The results confirmed that there is a reduction in the spectral efficiency when compared to the best path selection strategy due to the selection of a relay with bad channel condition. Including the controlling parameter in the expression of the spectral efficiency gives the designer or operator of wireless networks the capability to manage the amount of resources that can be used and the resulting efficiency.

\section{REFERENCES}

[1] H. Wang, S. Ma, and T. S. Ng "'On performance of cooperative communication systems with spatial random relays," IEEE Trans. on Commun., vol. 59, no. 4, pp. 1190-1199, 2011, doi: 10.1109/TCOMM.2011.012711.090285A.

[2] Q. Li, R. Q. Hu, Y. Qian and G. Wu, "Cooperative communications for wireless networks: techniques and applications in LTE-advanced systems," IEEE Wireless Communications, vol. 19, no. 2, pp. 22-29, 2012, doi: 10.1109/MWC.2012.6189409.

[3] X. Liang, M. Chen, I. Balasingham, and V.C. Leung, "Cooperative communications with relay selection for wireless networks: design issues and applications,” Wirel. Commun. Mob. Comput., vol. 13, no. 8, pp. 745-759, 2013, doi: $10.1002 / \mathrm{wcm} .1138$.

[4] G. Ke, G. Qiang, F. Li, and X. Huagang, "Relay selection in cooperative communication systems over continuous time-varying fading channel," Chin. J. Aero. vol. 30, no. 1, pp. 754-762, 2016, doi: 10.1016/j.cja.2016.12.021.

[5] M. Asshad, S.A. Khan, A. Kavak, K. Küçük, and D.L. Msongaleli, "Cooperative communications using relay nodes for next-generation wireless networks with optimal selection techniques: A review," IEEJ Trans Elec Electron Eng, vol. 14, no. 15, pp. 658-669, 2019, doi: 10.1002/tee.22852.

[6] D-T. Do and M-S V. Nguyen, "Enabling relay selection in non-orthogonal multiple access networks: direct and relaying mode," TELKOMNIKA Telecommunication Computing Electronics Control, vol. 18, no. 2, pp. 587-594, 2020, doi: 10.12928/telkomnika.v18i2.13313.

[7] E. S. Altubaishi and X. Shen, "Variable-rate based relay selection scheme for decode-and-forward cooperative networks," 2011 IEEE Wireless Communications and Networking Conference, pp. 1887-1891, 2011, doi: 10.1109/WCNC.2011.5779421.

[8] A. Bletsas, A. Khisti, D.P. Reed, and A. Lippman, "A simple cooperative diversity method based on network path selection," IEEE Journal on Selected Areas in Communications, vol. 24, no. 3, pp. 659-672, 2006, doi: 10.1109/JSAC.2005.862417.

[9] E. S. Altubaishi and X. Shen, "Performance analysis of spectrally efficient amplify-and-forward opportunistic relaying scheme with adaptive modulation," Wireless Commun. and Mobile Computing (Wiley), vol. 15, no. 8, pp. 1247-1258, 2015, doi: 10.1002/wcm.2397. 
[10] Z. Zhou, S. Zhou, S. Cui, and J. Cui, "Energy-efficient cooperative communication in clustered wireless sensor networks," IEEE Trans. Veh. Technol., vol. 57, no. 6, pp. 3618-3628, Nov. 2008, doi: 10.1109/TVT.2008.918730.

[11] X. Chen, Q. F. Zhou, T.-w. Siu, and F. C. M. Lau, "Asymptotic analysis of opportunistic relaying based on the max-generalized-mean selection criterion," IEEE Trans. Wireless commun., vol. 10, no. 4, pp. 1050-1057, 2011, doi: 10.1109/TWC.2011.012411.101426.

[12] K. Tourki, H.-C. Yang, and M.-S. Alouini, "Accurate outage analysis of incremental decode-and-forward opportunistic relaying," IEEE Trans. Wireless commun., vol. 10, no. 4, pp. 1021-1025, 2011, doi: 10.1109/TWC.2011.021611.100472.

[13] H. Cui, G. Wei, and Y. Wang, "Effects of CSI on ASEP based opportunistic DF relaying systems," IEEE Trans. Veh. Technol., vol. 60, no. 4, pp. 1898-1904, 2011, doi: 10.1109/TVT.2011.2131694.

[14] L. Dai, W. Chen, L.J. Cimini, and K.B. Letaief, "Fairness improves throughput in energy-constrained cooperative Ad-Hoc networks," IEEE Trans. Wireless Commun., vol. 8, no. 7, pp. 3679-3691, 2009, doi: 10.1109/TWC.2009.080856.

[15] Z-C Wang, Y.-Q Cao, T. An, and C.-Y. Zhu, "An opportunistic relaying selection scheme based on relay fairness," Indonesian Journal of Electrical Engineering, vol. 12, no. 1, pp. 218-224, 2014, doi: 10.11591/telkomnika.v12i1.3910.

[16] N. Nomikos, T. Charalambous, I. Krikidis, D. N. Skoutas, D. Vouyioukas and M. Johansson, “A buffer-aided successive opportunistic relay selection scheme with power adaptation and inter-relay interference cancellation for cooperative diversity systems," in IEEE Transactions on Communications, vol. 63, no. 5, pp. 1623-1634, 2015, doi: 10.1109/TCOMM.2015.2416234.

[17] N. Nomikos, D. Vouyioukas, T. Charalambous, I. Krikidis, P. Makris, D.N. Skoutas, M. Johansson, and C. Skianis, "Joint relay-pair selection for buffer-aided successive opportunistic relaying," Trans. Emerging Tel. Tech., vol. 25, no. 8, pp. 823-834, 2014, doi: 10.1002/ett.2718.

[18] T. Song, W. Kim, and S. Pack, "ORS-FA: An opportunistic relay selection scheme for frame-aggregated environments," Wireless Personal Communications, vol. 82, pp. 2351-2361, 2015, doi: 10.1007/s11277-015-23510 .

[19] J. Wang and Q. Wu, "Relay selection for maximizing the number of successive transmission in cooperative networks," International Journal of Parallel, Emergent and Distributed Systems, vol. 32, no. 6, pp. 632-646, 2017 doi: 10.1080/17445760.2016.1157179.

[20] I. Raziah, Y. Away, and N. Nasaruddin, "An adaptive best relay selection for energy efficient cooperative D2D Communications," 2019 International Conference on Radar, Antenna, Microwave, Electronics, and Telecommunications (ICRAMET), pp. 69-73, 2019, doi: 10.1109/ICRAMET47453.2019.8980433.

[21] P. Mach, Z. Becvar, and T. Spyropoulos, "Incentive mechanism and relay selection for D2D relaying in cellular networks," 2019 IEEE Global Communications Conference (GLOBECOM), pp. 1-7, doi: 10.1109/GLOBECOM38437.2019.9013205, 2019.

[22] Ahmed alkhayyat, Kassem Al Attabi, Qammer h. Abbasi, "Single relay selection in the cognitive cooperative network: toward bandwidth efficiency improvement," 2019 4th Scientific International Conference Najaf (SICN), pp. 222-226, 2019, doi: 10.1109/SICN47020.2019.9019350.

[23] R. Zhang, R. Nakai, K. Sezaki, and S. Sugiura, "Generalized bufferstate-based relay selection in cooperative cognitive radio networks," IEEE Access, vol. 8, pp. 11644-11657, 2020, doi: 10.1109/ACCESS.2020.2965742.

[24] F. L. Duarte and R. C. de Lamare, "Switched max-link relay selection based on maximum minimum distance for cooperative MIMO Systems," IEEE Transactions on Vehicular Technology, vol. 69, no. 2, pp. 1928-1941, 2020, doi: 10.1109/TVT.2019.2962533.

[25] A.J. Goldsmith, Wireless communications, Cambridge: Cambridge University Press, 2005. 